\title{
ON THE PROBLEM OF VIBRATION PROTECTION OF ROTOR SYSTEMS WITH ELASTIC ADAPTIVE ELEMENTS OF QUASI-ZERO STIFFNESS
}

\author{
Volodymyr KLITNOI, Anatoliy GAYDAMAKA \\ National Technical University «Kharkiv Polytechnic Institute», Department of Machine Components and \\ Mechatronic Systems, 2, Kyrpychova str., 61002, Kharkiv, Ukraine \\ e-mail: vladklitnoi@gmail.com \\ Abstract \\ The analysis of scientific publications for rotor systems on the elastic supports made it possible to \\ develop a basic version of the design scheme of active elastic support with controlled quasi-zero stiffness \\ based on adaptive piezoceramic elements. The main components of the mathematical model of the \\ functioning of active elastic supports with controlled quasi-zero stiffness based on adaptive piezoceramic \\ elements are substantiated, which will help create the foundations of a theory for solving the problem of \\ effective vibration protection. Numerical results show the possibility of effective applying of the proposed \\ solution to the problem of rotor vibration damping.
}

Keywords: rotor systems, supports with quasi-zero stiffness, adaptive materials, piezoceramics

\section{INTRODUCTION}

Improving the efficiency of rotor systems while reducing their mass and dimensions is connected to the need to increase the rotor speed. The operating rotational speeds of the rotor systems of many modern machines are higher than the first natural frequencies. Therefore, during acceleration and braking, the rotor passes through several resonances, accompanied by significant dynamic loads, which can lead to the destruction of the machine. Besides the unwanted vibration caused by mass unbalance and asymmetry, is inevitable in real systems.

Despite the development of modern rotor balancing methods $[1,2]$ in the process of balancing it is almost impossible to achieve complete balance of the rotor.

For reducing bearings overload and amplitudes of oscillations at operating speeds and when passing through critical ones is using elastic supports. Such supports with an undeniable advantage in the form of simplicity of design, are not able to instantly and within the necessary limits change the stiffness when the rotor passes through resonance.

The transition of the rotor through resonance with an instantaneous change in stiffness and minimization of vibration imbalance at operating speeds can be achieved by using elastic supports with quasi-zero stiffness. However, the efficiency of such systems is directly related to the solution of the issue of their configuration. The low stiffness of the elastic element requires a fairly accurate adjustment to the design load: a change in the magnitude of this load will lead to a loss in the functional reliability of the system. Consequently, there is a need to develop elastic vibration protection systems that allow their automatic adjustment for a given range of changes of the external load on the rotor bearings, that is, systems with controlled stiffness.

\section{LITERATURE ANALYSIS AND PROBLEM STATEMENT}

Known constructions of rotor elastic support systems can be divided into two classes: supports with elastic elements that maintain a stability form and supports with elastic elements of quasi-zero stiffness. The first one includes the squeeze film dampers [3]. The authors note that squeeze film dampers have highly nonlinear characteristics and are more complex to design and analyze than other machinery elements. For better performance in reducing nonlinear characteristics elastic ring placing inside the oil chamber [4]. In [5] provides guidelines for the squeeze film dampers design in an auxiliary bearing application. Other well-known elastic support - squirrel-cage is widely used as an energy absorption component [6, 7]. In [8] represented design of squirrel cage considering the rotor system stability, dynamical response and squeeze film dampers damping effect. Several works can be found in the literature, with the purpose of modeling rotors mounted either on viscoelastic materials or on bearings made of this type of material. So, in [9] was developed a methodology for the design of angular viscoelastic dynamic vibration neutralizer to control rotor flexural vibration. In [10] was proposed the support characterized by adding a discrete tuning mass to 
the system attached by means of two viscoelastic sheets characterizing double isolation in the system.

The considered supports with elastic elements that maintain the stability form have not solve the problem of reducing vibration activity completely, since they are not able to instantly and within the necessary limits change the stiffness when passing through resonance.

The transition of the rotor through resonance with an instantaneous change in stiffness can be achieved by using elastic systems with quasi-zero stiffness. The idea of the functioning of systems with quasi-zero stiffness based on the "Mises farm" for vibration protection of dynamic objects was first investigated by Alabuzhev [11]. If vibrations with a certain predetermined amplitude occur in the system, then when tuning the system to an external load, a section with quasi-zero stiffness appears. In this case, the damping object will always be in a transient oscillatory process, the amplitude of which will be limited. In the passive support of bearings with quasi-zero stiffness [12], the membrane is an elastic element. When the rotor system passes through resonance under the influence of a sharply increasing load on the support, "clicking" occurs and the membrane takes on a new form of equilibrium. The efficiency of the proposed passive support is directly related to the issues of its adjustment. As a rule, a sufficiently accurate adjustment to the design load is required, otherwise, a loss of the functional reliability of the system and a decrease in operating efficiency are possible. This disadvantage of passive systems can be eliminated by the use of elastic supports with quasi-zero stiffness, which allow their adjustment to different loads - active supports.

Compared with the passive methods, the active vibration control systems are more complex and effective. Many methods of active vibration control have been proposed. In [13] was showed that active bearings can eliminate both resonances and bearing forces for a Jeffcott Rotor system. Active magnetic bearings are increasingly used in rotary technology due to their undeniable advantages, such as nolubrication and no-wear. In [14] use a flexible rotor supported on active magnetic bearings and the feedback control for stabilizing the system with remarkably mitigated rotor vibrations. A compensation controller is proposed for a rotor system with active magnetic bearings, effective to control imbalance vibration and improve accuracy [15]. Controlled magnetorheological squeeze-film dampers effective to reduce vibration and control the critical speeds of rotor systems were studied in [16]. The authors concentrating on determining the distribution of magnetic induction in the lubricating layer and calculating the damping forces. Active quasi-zero stiffness support was proposed in [17]. A microswitch with a time relay is used, which monitors the external load mode and by switching on the operation of the electromagnet prolongs the action of quasi-zero stiffness. The disadvantage of this support is the complexity and sufficiently large dimensions of the structure, the presence of a microswitch with a time relay and a delay in the operation of the structure narrows the possibilities of using automatic control circuits. For the further development of the proposed active quasi-zero stiffness system, the use of adaptive elements seems promising.

In the 1990s, authors investigated active vibration control of an air turbine with a piezohydraulic actuator system [18]. Significant reductions in vibration were achieved by the proposed system based on piezoelectric actuators. In [19] was studied flexible shaft supported with conventional ball bearings in combination with piezoactuators and force sensors. The experiments showed a significant reduce in the bearing forces and displacements in the active case. The vibration isolation to minimize forces caused by unbalance through the application of piezoelectric actuators in combination with the fxlms-algorithm is shown in [20]. The suppression of rotor instability supported by sliding journal bearings by actively controlling vibration using piezo actuators was studied in [21]. The bearing bushing was being moved by means of two piezoactuators oriented in vertical and horizontal directions. The shaft rotational velocity, at which occurs the onset of instability, was substantially increased. In [22] a ring-shaped piezoelectric damper, which uses shunted piezoelectric stack has been proposed. The results of studies show that this damper may be effective for vibration control within wide frequency band. All represented works make us realize the great potential of using piezoelectric actuators in active quasi-zero stiffness support to suppress vibration of rotor systems.

The analysis of the considered structures showed that supports with elastic elements that maintain a stability form are not able to instantly and within the necessary limits change the stiffness when the system passes through resonance. The effectiveness of vibration protection systems with elastic elements, which have two stable equilibrium positions, is largely determined by their adjustment. It is proposed that automatic tuning to a given range of load changes in vibration protection systems with quasi-zero stiffness be made using adaptive piezoelectric elements.

\section{PURPOSE AND OBJECTIVE OF THE STUDY}

The aim of the work is to substantiate the main directions of theoretical research to solve the problem of vibration protection of rotor systems through the use of active elastic supports with controlled quasi-zero stiffness based on adaptive elements.

To achieve this goal, it is necessary to solve the following tasks: 
- offer a typical variant of the structural scheme of elastic support with controlled quasi-zero stiffness based on adaptive elements;

- substantiate the main components of a mathematical model of the functioning of elastic supports with controlled quasi-zero stiffness based on adaptive elements;

- evaluate the performance of the proposed mathematical model of elastic support by numerical studies.

\section{DEVELOPMENT OF THE STRUCTURAL SCHEME OF ELASTIC SUPPORT WITH ADAPTIVE ELEMENTS OF QUASI-ZERO STIFFNESS}

Figure 1 presents the basic model of the support of rotor systems with a central active elastic element and a control elastic element made of adaptive material and an active control system [23]. When selecting a material for performing adaptive elements, it is possible to distinguish materials that directly convert the externally applied electric effect into a mechanical one, or, conversely, turn a mechanical effect into electric by a direct physical effect at the microstructural level. Among a large class of materials for the manufacture of active and control elastic support elements, the most promising today is piezoelectric ceramics. Its high integrability and a wide operating frequency range are the most suitable properties for using in active vibration control systems.

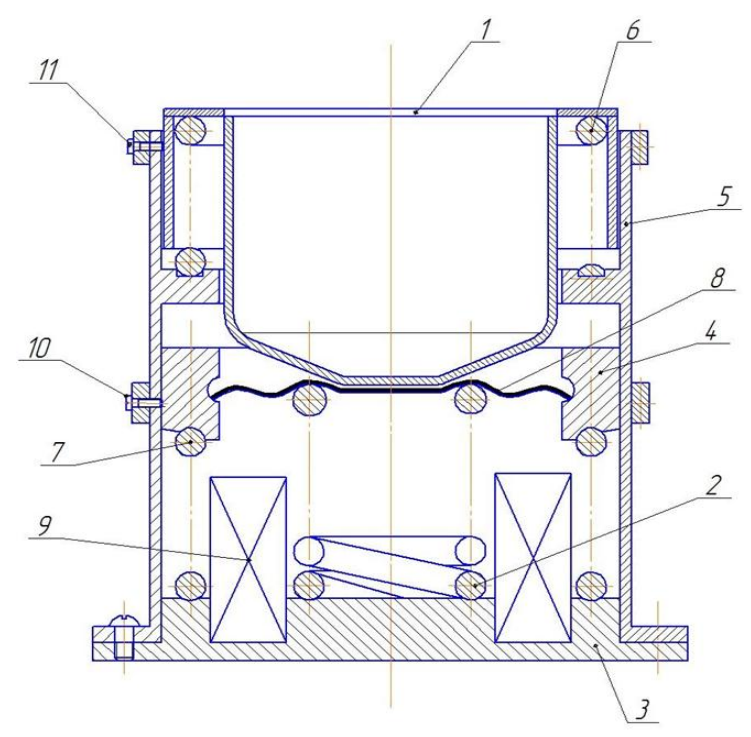

Fig. 1. Model of support with elastic adaptive elements of quasi-zero stiffness: 1 - central platform; 2 - central active elastic element; 3 supporting part; 4 - controller in the form of two symmetrically located sliders; 5 - guides; 6 - lateral elastic element; 7 - control elastic element; 8 - elastic element in the form of a conical corrugated shell; 9 - active control system; 10,11 - set screws
The proposed vibration protection system with actively controlled quasi-zero stiffness works as follows. With increasing load, the inertial sliders 4 are displaced along the guides 5 , the central active elastic element 2 together with the conical corrugated shell 8 , the lateral elastic element 6 , and the control elastic element 7 are deformed. In a certain mutual position of the elastic elements, the system instantly changes its stiffness, and, therefore, changes the range of load frequencies it perceives. The frequency range is controlled by the active control system 8 , due to which the deformation of the control element 7 is monitored and the necessary stiffness of the active element 2 is actively adjusted, thereby continuing the action of the quasi-zero stiffness of the support.

The proposed support solves the task of vibration protection of rotor systems by monitoring the response of the system to external influences using an auxiliary control element and changing, with the help of a control algorithm, the elastic properties of the central active element.

\section{MATHEMATICAL MODELS OF ELASTIC SUPPORT WITH ADAPTIVE PIEZOCERAMIC ELEMENTS OF QUASI- ZERO STIFFNESS}

An analytical approach to solving the problem of vibration protection of rotor systems with elastic adaptive elements of quasi-zero stiffness involves the development of the foundations of the theory of their functioning on the basis of a set of mathematical models for describing the dynamics of the vibration protection system, the electromechanical state and electrodynamics of active and control elastic elements.

The proposed model of elastic supports with adaptive elements of quasi-zero stiffness can be described by the scheme [24] (fig. 2).

Kinetic energy of the system

$$
T=\frac{m_{1} \dot{x}_{1}^{2}}{2}+m_{2} \dot{x}_{2}^{2},
$$

system potential energy

$$
\begin{aligned}
& \Pi=\frac{C_{10} x_{1}^{2}}{2}+C_{20} x_{2}^{2}+ \\
& +C_{12}\left(x_{2}-x_{1}\right)^{2}+C_{\text {п }}\left(h_{0}-h\right)^{2} .
\end{aligned}
$$

From the Lagrange equations of the 2nd kind and considering that the friction force $F_{f}=C_{\text {п }}\left(h_{0}-h\right) \cdot \frac{a}{h} \cdot f$, the equation of motion of support with elastic adaptive elements of quasi-zero stiffness 


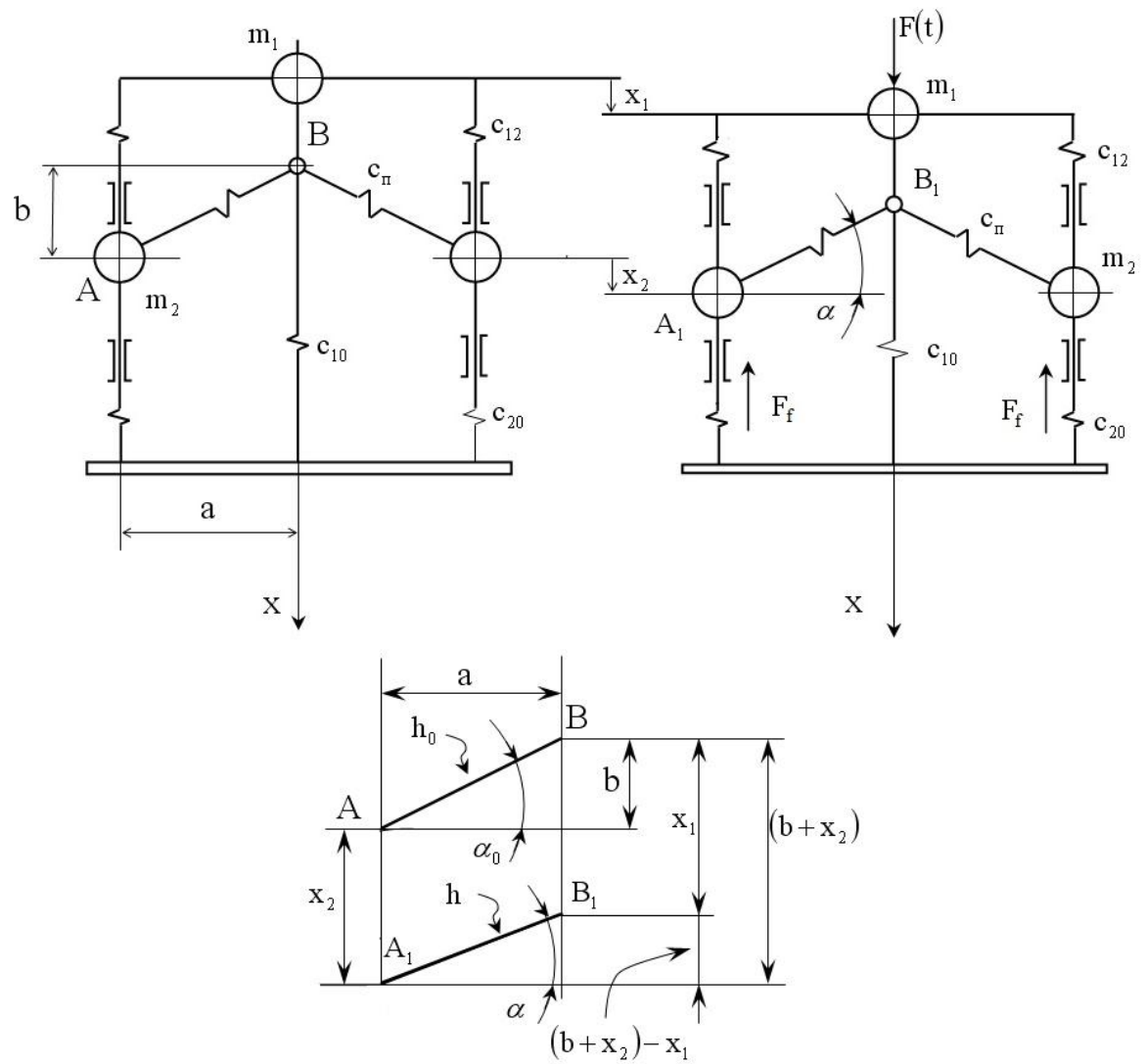

Fig. 2. Elastic support scheme, where $m_{1}, m_{2}$ - masses of retained system and sliders, respectively; $C_{10}, C_{12}, C_{20}, C_{\mathrm{n}}-$ stiffnesses of the elastic elements of the system; $h_{0}, h, b, a, \alpha$-geometric parameters of the system; $x_{1}, x_{2}-$ displacements of the points of the system; $F(t)$ - external force; $F_{f}$-friction force in the guides.

$$
\left.\begin{array}{l}
m_{1} \ddot{x}_{1}+C_{10} x_{1}-2 C_{12}\left(x_{2}-x_{1}\right)+ \\
+2 C_{\text {п }}\left(\frac{h_{0}}{h}-1\right)\left(b+x_{2}-x_{1}\right)=F(t), \\
m_{2} \ddot{x}_{2}+C_{20} x_{2}+C_{12}\left(x_{2}-x_{1}\right)- \\
-C_{\text {п }}\left(\frac{h_{0}}{h}-1\right)\left(b+x_{2}-x_{1}-a f\right)=0,
\end{array}\right\}
$$

$f$ - coefficient of friction in the guides.

Denoting $Z=x_{1}-x_{2}$ can get the static equation

$$
\begin{aligned}
& \frac{F}{C_{10}}=\left(1+C_{12} \frac{C_{10}+2 C_{20}}{C_{10} C_{20}}\right) Z+ \\
& +C_{\text {п }}\left(\frac{h_{0}}{h}-1\right)\left[\frac{C_{10}+2 C_{20}}{C_{10} C_{20}}(b-Z)-\frac{a}{C_{20}} f\right] ; \\
& h=\sqrt{a^{2}+(b-Z)^{2}} .
\end{aligned}
$$

Using dimensionless parameters $\quad \bar{x}_{1}=\frac{x_{1}}{b}$, $\bar{x}_{2}=\frac{x_{2}}{b}, \quad \bar{Z}=\frac{Z}{b}, \quad \bar{h}=\sqrt{\bar{h}_{0}^{2}-2 \bar{Z}+\bar{Z}^{2}}, \quad$ system stiffness can be represented as:

$$
\begin{aligned}
& \left(b \cdot C_{10}\right)^{-1} \frac{d F}{d \bar{Z}}=1+C_{12} \cdot \pi_{c}+ \\
& +C_{\text {п }}\left[\begin{array}{l}
\frac{\bar{h}_{0}(1-\bar{Z})}{\bar{h}^{3}}\left(\pi_{c}-(1-\bar{Z})-\pi_{f} \frac{2}{C_{10}}\right)- \\
-\left(\frac{\bar{h}_{0}}{\bar{h}}-1\right) \pi_{c}
\end{array}\right], \\
& \pi_{c}=\frac{C_{10}+2 C_{20}}{C_{10} C_{20}}, \quad \pi_{f}=\frac{f}{C_{10} \operatorname{tg} \alpha_{0}} .
\end{aligned}
$$

The obtained dependences (4), (5) based on the development of equations (3), taking into account the design features of an elastic support with controlled quasi-zero stiffness, will allow us to calculate the input signal to the control adaptive element from the range of external influence and predict the necessary stiffness of the active element.

Electromechanical equations [25, 26]:

$$
\begin{aligned}
& \sigma=\mathbf{C} \cdot \varepsilon-\mathbf{e} \cdot \mathbf{E}, \\
& \left.\mathbf{D}=\mathbf{e}^{\mathbf{T}} \cdot \varepsilon+\mathbf{c}^{\varepsilon} \cdot \mathbf{E},\right\} \\
& \varepsilon=\mathbf{S} \cdot \sigma+\mathbf{d}^{\mathbf{T}} \cdot \mathbf{E}, \\
& \mathbf{D}=\mathbf{d} \cdot \sigma+\mathbf{c}^{\sigma} \cdot \mathbf{E},
\end{aligned}
$$

$\sigma$ - stress tensor; $\varepsilon$ - strain tensor; D - electric induction vector; $\mathbf{E}$ - electric field vector; $\mathbf{C}$ tensor of elastic stiffness coefficients at constant electric field strength; $\mathbf{S}-$ tensor of elastic 
compliance coefficients at constant electric field strength; $\mathbf{\epsilon}^{\varepsilon}, \mathbf{\epsilon}^{\sigma}$ - tensors of absolute dielectric constant coefficients at constant mechanical deformation and constant mechanical stress, respectively; e - piezoelectric constant tensor; d piezoelectric coefficient tensor. After finding their components, taking into account the properties of the piezoelectric materials, the factors of deformation uniformity, stress field uniformity it will allow constructing a system of equations describing the electro-mechanical models of active and controlling elastic elements made of piezoelectric ceramics.

In addition to equations of state, the equations of motion and electrodynamics must be included in the system. The equations of motion for piezoceramics can be represented in the following form $[25,26]$ :

$$
\mathbf{D}_{u}^{T}=\left[\begin{array}{cccccc}
\frac{\partial}{\partial x_{1}} & 0 & 0 & \frac{\partial}{\partial x_{2}} & 0 & \frac{\partial}{\partial x_{3}} \\
0 & \frac{\partial}{\partial x_{2}} & 0 & \frac{\partial}{\partial x_{1}} & \frac{\partial}{\partial x_{3}} & 0 \\
0 & 0 & \frac{\partial}{\partial x_{3}} & 0 & \frac{\partial}{\partial x_{2}} & \frac{\partial}{\partial x_{1}}
\end{array}\right]
$$

$\mathbf{X}$ - volumetric forces vector; $\mathbf{u}$ - vector of mechanical displacements; $\rho$ - mass density; $\mathbf{D}_{u}^{T}$ - differentiation matrix.

Due to the fact that piezoelectric effects can be arbitrarily called quasi-mechanical, since they are associated with velocities much lower than the speed of light in a dielectric, magnetic effects can be neglected and electrostatic equations can be considered instead of electrodynamics equations [26]. With this in mind, we can use the well-known Maxwell equations

$$
\begin{gathered}
\mathbf{D}_{\varphi}^{T} \mathbf{D}=0 \\
\mathbf{D}_{\varphi}^{T}=\left[\begin{array}{lll}
\frac{\partial}{\partial x_{1}} & \frac{\partial}{\partial x_{2}} & \frac{\partial}{\partial x_{3}}
\end{array}\right]
\end{gathered}
$$

$\mathbf{D}_{\varphi}^{T}$ - differentiation matrix.

Equations (4-7) supplemented by the equations of coupling between the electric field vector $\mathbf{E}$ and the electric potential

$$
\mathbf{E}=-\mathbf{D}_{\varphi} \phi
$$

and equations of coupling between the strain vector and mechanical displacements

$$
\varepsilon=\mathbf{D}_{u} \mathbf{u}
$$

form a closed system of differential equations of the electromechanical model of the active and control elastic piezoceramic elements. Supplementing these equations with boundary conditions selected depending on the design features of the elastic support with adaptive quasi-zero stiffness and initial conditions determined using equations (3-5), we can arrive at initial boundary-value problems, which are the basis for modelling of active and controlling elastic piezoceramic elements. The solution of such a mathematical model allows us to evaluate the response of the controlling piezoceramic elements to the input external mechanical action and the reaction of the active element to the electrical effect.

\section{NUMERICAL ANALYSIS}

The rotor system supported by 2 bearings with adaptive quasi-zero stiffness was considered as a calculation model (fig. 3). The rotor was assumed to be rigid and symmetrical.

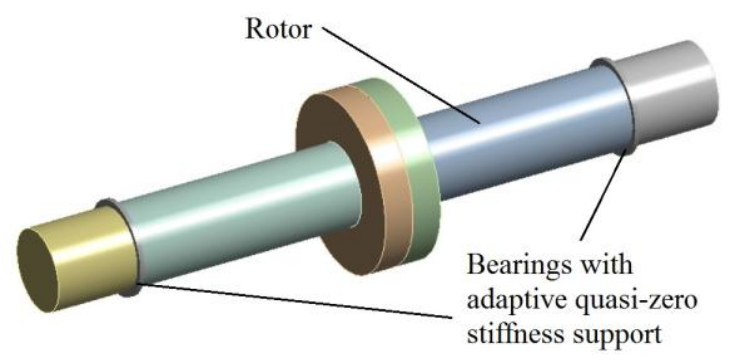

Fig. 3. Three-dimension model of a rotor system with adaptive quasi-zero stiffness supports.

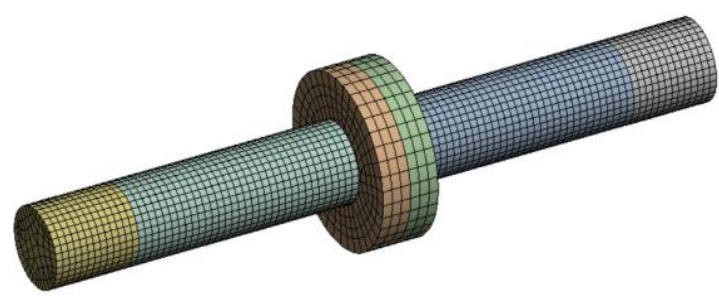

Fig. 4. FEM model of a rotor system.

The radial amplitude of the rotor was calculated by the finite element method. The sweep method was applied to mesh the three-dimension model (fig. 4).

The interaction between the control and active piezoelectric elements was implemented using the algorithm of a direct velocity feedback control scheme [27, 28].

The radial vibration in frequency domain is shown in fig. 5. The resonance is clearly visible in the uncontrolled case. In controlled case amplitude peaks are eliminated.

\section{DISCUSSION}

The concept of using adaptive materials in vibroprotective systems with controlled quasi-zero stiffness is very promising. Piezoelectric ceramics selected as a material for adaptive elements is characterized by high noise immunity and a low level of intrinsic noise. Ceramic technology for the manufacture of piezoelectric elements does not impose fundamental restrictions on their shape and size. These circumstances, as well as the high values of the piezoelectric characteristics, 


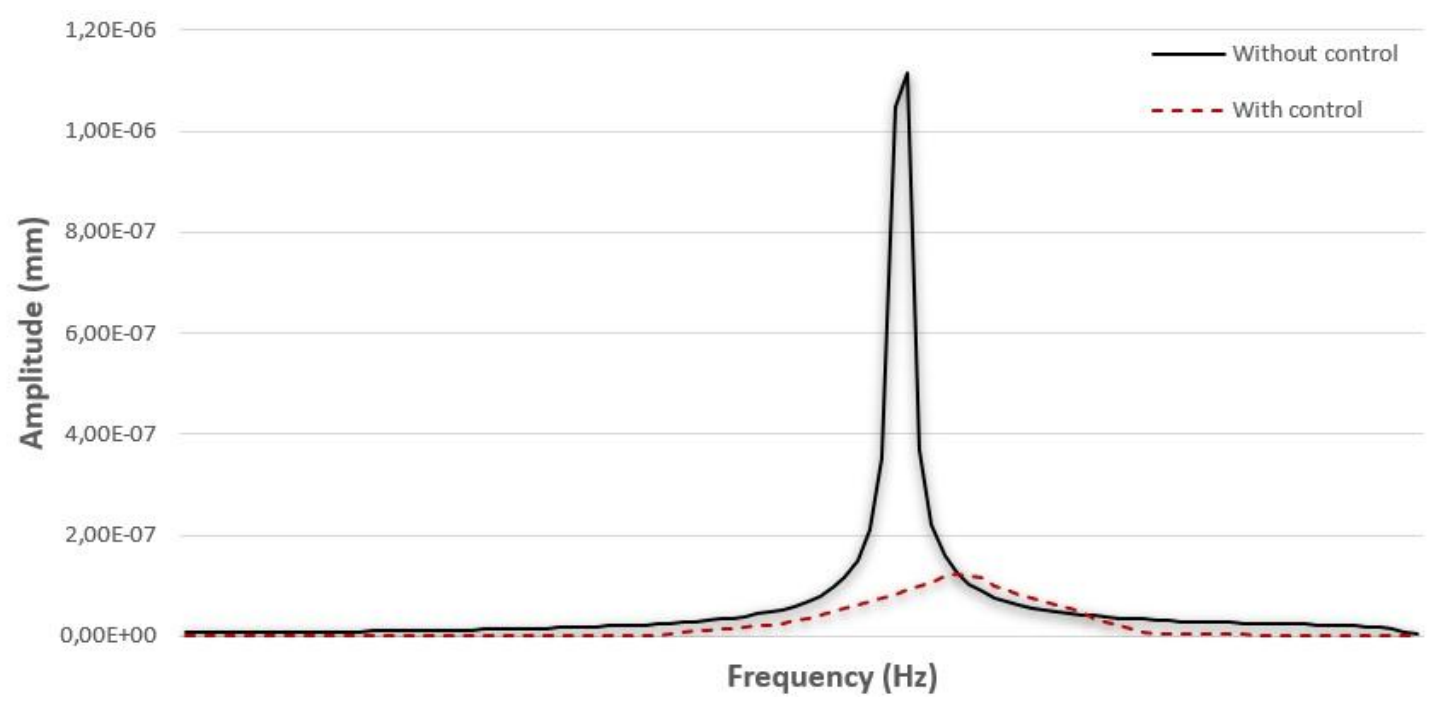

Fig. 5. Radial vibration in frequency domain.

determine the effective use of ceramic piezoelectric elements in vibration protection systems with controlled quasi-zero stiffness as control and active elements.

An important advantage of systems based on piezoceramic elements is also a fairly low energy dependence (the ability to design an energy system that does not require external energy consumption using control piezoceramic elements as energy sources).

Specific values of the design parameters of elastic supports with controlled quasi-zero stiffness in the form of adaptive piezoceramic elements can be obtained based on numerical methods.

In some cases, it is advisable to simplify the presented basic mathematical model by moving from the original three-dimensional electromechanical model to the one-dimensional problem.

Despite the good results obtained by the numerical studies with using a direct velocity feedback control scheme, to implement the most stable and effective interaction between control and active adaptive elements, it is necessary, based on the results of a comprehensive analysis, to choose the most optimal active control scheme.

\section{CONCLUSIONS}

The concept of improving vibration protection systems with controlled quasi-zero stiffness through the use of adaptive elements is proposed. A basic version of the design scheme of the active vibration protection system with adaptive elements has been developed. Piezoelectric ceramics are used as material for adaptive elements.

The main components of the mathematical model of elastic supports with controlled quasi-zero stiffness are substantiated for the theoretical foundations of solving the problem of effective vibration protection of rotor systems, taking into account the reactions and responses of piezoceramic elements.

The validity of the proposed model is confirmed by numerical studies.

\section{REFERENCES}

1. Zachwieja J. The effectiveness of modal balancing of flexible rotors. Diagnostyka. 2015;16(3):79-87.

2. Amroune S, Belaadi A, Menasri N, Zaoui M, Mohamad B, Amin H. New approach for computeraided static balancing of turbines rotors. Diagnostyka. 2019;20(4):95-101. https://doi.org/10.29354/diag/114621

3. Zeidan FY, Andres LS, Vance JM. Design and application of squeeze film dampers in rotating machinery. Texas A\&M University. Turbomachinery Laboratories. 1996;169-188. https://doi.org/10.21423/R1694R

4. Zhifei H, Qian D, Wei Z. Dynamical analysis of an elastic ring squeeze film damper-rotor system. Mechanism and Machine Theory. 2019;131: 406-419. https://doi.org/10.1016/j.mechmachtheory.2018.10.011

5. Xiao K, Palazzolo A, Wan Z. Auxiliary bearing squeeze film dampers for magnetic bearing supported rotors. Tribology International. 2020;146,106181. https://doi.org/10.1016/j.triboint.2020.106181

6. Qingkai H, Yugang C, Hao Z, Lingli J, Xuejun L. Vibrations of rigid rotor systems with misalignment on squirrel cage supports. Journal of Vibroengineering. 2016;18,7: 4329- 4339. https://doi.org/10.21595/jve.2016.16860

7. Mao Y, Wang L, Zhang C. Study on the load distribution and dynamic characteristics of a thinwalled integrated squirrel-cage supporting roller bearing. Appl. Sci. 2016;6,415. https://doi.org/10.3390/app6120415

8. Wei Z, Bingbing H, Xiang L, Jianqiao S, Qian D. Multiple-objective design optimization of squirrel cage for squeeze film damper by using cell mapping method and experimental validation. Mechanism and Machine Theory. 2019;132: 66-79.

https://doi.org/10.1016/j.mechmachtheory.2018.10.010

9. Voltolini, DR, Kluthcovsky S, Filho FD, Lopes E, Bavastri C. Optimal design of a viscoelastic vibration 
neutralizer for rotating systems: Flexural control by slope degree of freedom. Journal of Vibration and Control. 2018;24: 3525-3537. https://doi.org/10.1177/1077546317752797

10. Ribeiro EA, Pereira JT, Bavastri CA. Passive vibration control in rotor dynamics: Optimization of composed support using viscoelastic materials. Journal of Sound and Vibration. 2015;351: 43-56. https://doi.org/10.1016/j.jsv.2015.04.007

11. Alabuzhev P, Gritchin A, Kim L, Migirenko G, Chon V, Stepanov P. Vibration protecting and measuring systems with quasi-zero stiffness. Hemisphere Publishing, Taylor \& Francis Group, New York; 1989.

12. Gaponov VS, Gaydamaka AV, Naumov OI. Vibration protection system with controlled quasizero stiffness. Ukraine, Patent number 69042, 2012. Ukrainian.

13. Heindel S, Becker F, Rinderknecht S. Unbalance and resonance elimination with active bearings on a Jeffcott Rotor. Mechanical Systems and Signal Processing. 2017;85: 339-353. https://doi.org/10.1016/j.ymssp.2016.08.016

14. Zhao J, Zhang H, Fan M, Wu Y, Zhao H. Control of a constrained flexible rotor on active magnetic bearings. IFAC-PapersOnLine. 2015;48(28): 156161. https://dx.doi.org/10.1016/j.ifacol.2015.12.117

15. Yao X, Chen Z, Jiao Y. Unbalance vibration compensation control using deep network for rotor system with active magnetic bearings. Mechanisms and Machine Science 2019;60: 72-81. https://doi.org/10.1007/978-3-319-99262-4_6

16. Ferfecki P, Zapoměl J, Kozánek J. Analysis of the vibration attenuation of rotors supported by magnetorheological squeeze film dampers as a multiphysical finite element problem. Advances in Engineering Software. 2017;104: 1-11. https://doi.org/10.1016/j.advengsoft.2016.11.001

17. Gaponov VS, Gaydamaka AV, Naumov OI. Active vibration protection system with automatic bearing supports control. Ukraine, Patent number 80416, 2013. Ukrainian.

18. Tang P, Palazzolo AB, Kascak AF, Montague GT. Active vibration control of rotating machinery with a hybrid piezohydraulic actuator system. J. Eng. Gas Turbines Power. 1995;117(4): 767-776. http://dx.doi.org/10.1115/1.2815463

19. Heindel S, Müller PC, Rinderknecht S. Unbalance and resonance elimination with active bearings on general rotors. Journal of Sound and Vibration. 2018;431: 422-440. https://doi.org/10.1016/j.jsv.2017.07.048

20. Hasch B, Lindenborn O, Nordmann R, Ulbrich H, Ginzinger L. Model-based fault detection on a rotor in an actively supported bearing using piezoelectric actuators and the fxlms-algorithm. Motion and Vibration Control. 2009; 123-132. https://doi.org/10.1007/978-1-4020-9438-5_13

21. Tuma J, Šimek J, Škuta J, Los J. Active vibrations control of journal bearings with the use of piezoactuators. Mechanical Systems and Signal Processing. 2013;36,2: 618-629.

https://doi.org/10.1016/j.ymssp.2012.11.010

22. Huan H, Xing T, Jincheng H, Fang Z, Guoping C. A novel ring-shaped vibration damper based on piezoelectric shunt damping: Theoretical analysis and experiments. Journal of Sound and Vibration. 2020; 468,115125 .

https://doi.org/10.1016/j.jsv.2019.115125

23. Gaydamaka AV, Klitnoi VV. Active vibration protection system with adaptive quasi-zero stiffness for rotor system bearing supports. Ukraine, Patent number 125538, 2017. Ukrainian.

24. Gaponov VS, Naumov OI, Ostapchuk YO. A mathematical model of an elastic support with controlled quasi-zero stiffness for high-speed rotary bearing systems. Bulletin of NTU "KhPI". Series: Energy and thermal processes and equipment. 2012; 8: 131-136. Russian.

25. Ikeda T. Fundamentals of piezoelectricity. Oxford University Press, New York; 1996.

26. Aronov BS. Electromechanical transducers of piezoelectric ceramics. Leningrad, Energoatomizdat; 1990. Russian.

27. Klitnoi VV. Numerical studies of vibration control of the on-board equipment printed unit. Bulletin of NTU "KhPI". 2008; 31: 77-83. Russian.

28. Preumont A. Vibration Control of Active Structures. Springer, Netherlands; 2011.

https://doi.org/10.1007/978-94-007-2033-6

Received 2020-03-15

Accepted 2020-05-18

Available online 2020-05-19

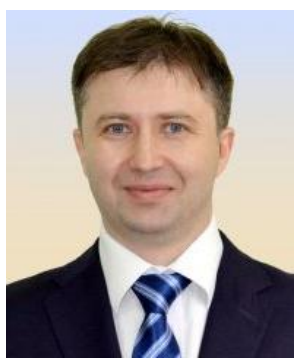

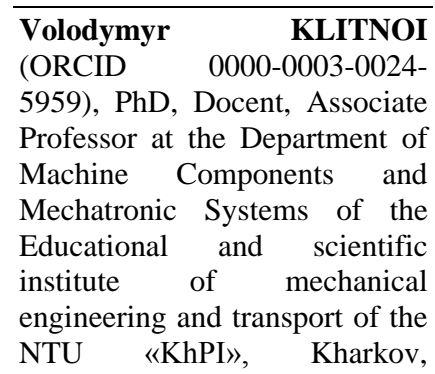
Ukraine. Main directions of scientific research: vibration isolation and vibration protection systems for units and machines, active vibration control systems.

e-mail: vladklitnoi@gmail.com

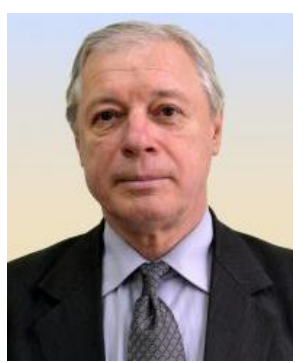

Anatoliy

GAYDAMAKA (ORCID 0000-0002-69524086), Doctor of Technical Sciences, Professor, Head of the Department of Machine Components and Mechatronic Systems of the Educational and scientific institute of mechanical engineering and transport of the NTU «KhPI», Kharkov, Ukraine. Main directions of scientific research: methods for improving the technical level of machine. e-mail: gaydamaka.doc@gmail.com 